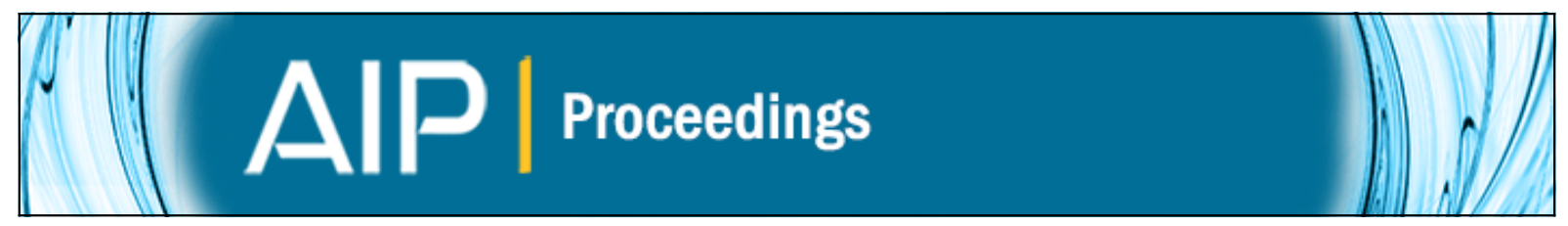

\title{
New Results from Bright Metal-Poor Stars in the Hamburg/ESO Survey
}

Anna Frebel, Timothy C. Beers, and Brian Marsteller

Citation: AIP Conference Proceedings 990, 113 (2008); doi: 10.1063/1.2905515

View online: http://dx.doi.org/10.1063/1.2905515

View Table of Contents: http://scitation.aip.org/content/aip/proceeding/aipcp/990?ver=pdfcov

Published by the AIP Publishing

\section{Articles you may be interested in}

The IMF of Extremely Metal-Poor Stars as Constrained by Data from the Milky Way

AIP Conf. Proc. 990, 462 (2008); 10.1063/1.2905665

Refined Estimates of Carbon Abundances for Carbon-Enhanced Metal-Poor Stars

AIP Conf. Proc. 990, 154 (2008); 10.1063/1.2905526

Revised Parameter Estimates For The Most Metal-Poor Candidates In SDSS-I And SEGUE

AIP Conf. Proc. 990, 151 (2008); 10.1063/1.2905525

The Giants Stars HE 0107-5240 and HE 0557-4840 and New Searches for Metal-Poor Stars

AIP Conf. Proc. 990, 109 (2008); 10.1063/1.2905514

Searches for the Most Metal-Poor Candidates from SDSS and SEGUE

AIP Conf. Proc. 990, 104 (2008); 10.1063/1.2905513 


\title{
New Results from Bright Metal-Poor Stars in the Hamburg/ESO Survey
}

\author{
Anna Frebel*, Timothy C. Beers ${ }^{\dagger}$ and Brian Marsteller ${ }^{\dagger}$ \\ ${ }^{*}$ McDonald Observatory, University of Texas, Austin, TX 78712-0259, USA \\ ${ }^{\dagger}$ Department of Physics \& Astronomy, CSCE: Center for Study of Cosmic Evolution, and JINA: Joint Institute \\ for Nuclear Astrophysics, Michigan State University, E. Lansing, MI 48824-1116, USA
}

\begin{abstract}
We present an abundance analysis of HE 1327-2326, currently the most iron-poor star known, based on a newly acquired VLT spectrum. The $1 \mathrm{D}$ abundance pattern is corrected for 3D effects. The 3D iron abundance is $[\mathrm{Fe} / \mathrm{H}]=-5.9$, while the $\mathrm{CNO}$ elements of the star are extremely overabundant $[\mathrm{CNO} / \mathrm{Fe}] \sim 3$ to 4 ). The cosmologically important element $\mathrm{Li}$ is still not detected; the new upper limit is $A(\mathrm{Li})<0.6$. A new analysis of the medium-resolution data of the sample of bright metal-poor stars from the Hamburg/ESO Survey (HES) was carried out. We are using this sample to obtain clues to the chemical nature of the early Universe by investigating the kinematic properties of the sample. Based on estimated $[\mathrm{Fe} / \mathrm{H}]$ and $[\mathrm{C} / \mathrm{Fe}]$, we are also able to use the sample to test a formation mechanism for low-mass metal-poor stars.
\end{abstract}

Keywords: $97.10 . \mathrm{Tk}, 97.10 . \mathrm{Wn}, 97.20 . \mathrm{Tr}, 98.35 . \mathrm{Gi}$

\section{INTRODUCTION}

Studying large samples of metal-poor stars in the Galaxy provides important clues to the chemical nature of the early Universe, to the formation of the elements, and to the major nucleosynthesis processes that were involved. Investigation of the sample of bright metal-poor stars from the Hamburg/ESO survey [1] has led to the discovery of HE 1327-2326, the currently most iron-poor star known [2,3]. Due to its chemically primitive nature, this object is very important for our understanding of the early Universe and the formation of the first low-mass stars. In this paper we present new abundance measurements of HE $1327-2326$ based on a new, very high-S/N VLT spectrum. Full details of the new analysis can be found in Frebel et al. [4]. Furthermore, in an attempt to derive more accurate iron and carbon abundances, we have re-analyzed the entire bright sample with the SEGUE Stellar Parameter Pipeline (SSPP; Lee et al. $[5,6]$. We report the results and provide an outlook on an ongoing kinematic analysis of the sample.

\section{UPDATE ON THE ABUNDANCES OF HE 1327-2326}

In the Subaru discovery spectrum, seven weak Fe I lines were detected [3]. The strongest line was measured to have an equivalent width of $6.8 \mathrm{~m} \AA$. As already reported in Frebel et al. [7], our new VLT data confirms the presence of six of the seven lines (one line is not covered). Additionally, we detect another four lines, bringing the total to $11 \mathrm{Fe} I$ lines from which the metallicity can be deduced. We find HE 1327-2326 to have a 1D LTE $\mathrm{Fe}$ abundance of $[\mathrm{Fe} / \mathrm{H}]=-5.7$, the same as previously reported [3]. In this work we are now applying 3D LTE correction to our ID LTE abundances. Within the LTE framework, the 3D correction for Fe I is negative ( $\sim-0.2$; Collet et al. [8]). Thus, the Fe I abundance of $\mathrm{HE} 1327-2326$ is lowered to $[\mathrm{Fe} / \mathrm{H}]=-5.9$. 3D nonLTE corrections are not known at present [9]. For completeness, we note that in previous works the measured Fe I abundance was corrected by +0.2 dex to account for ID non-LTE effects in Fe I.

In principle, $\mathrm{Fe}$ II lines should be the preferred $\mathrm{Fe}$ abundance indicator. However, the strongest Fe II lines are extremely weak, and no Fe II line could be detected in either the Subaru nor in the new VLT spectrum. The $3 \mathrm{D}$-corrected upper limit is $[\mathrm{Fe} \mathrm{II} / \mathrm{H}]<-5.4$.

\section{CNO elements}

Carbon, nitrogen, and oxygen abundances for HE 1327-2336 were derived by Aoki et al. [3] (C and $\mathrm{N}$; from the Subaru spectrum) and then by Frebel et al. [7] (C and O; from the VLT spectrum). For the latter 1D LTE analysis, corrections were suggested for all three elements to account for 3D model atmosphere effects. The estimated 3D corrections were mostly based on works described in Asplund [9] and references therein.

Compared to abundances from atomic lines, for abundances derived from molecular features there actually is no straightforward 3D correction (e.g., a simple abundance offset). Molecular features consist of many differ- 


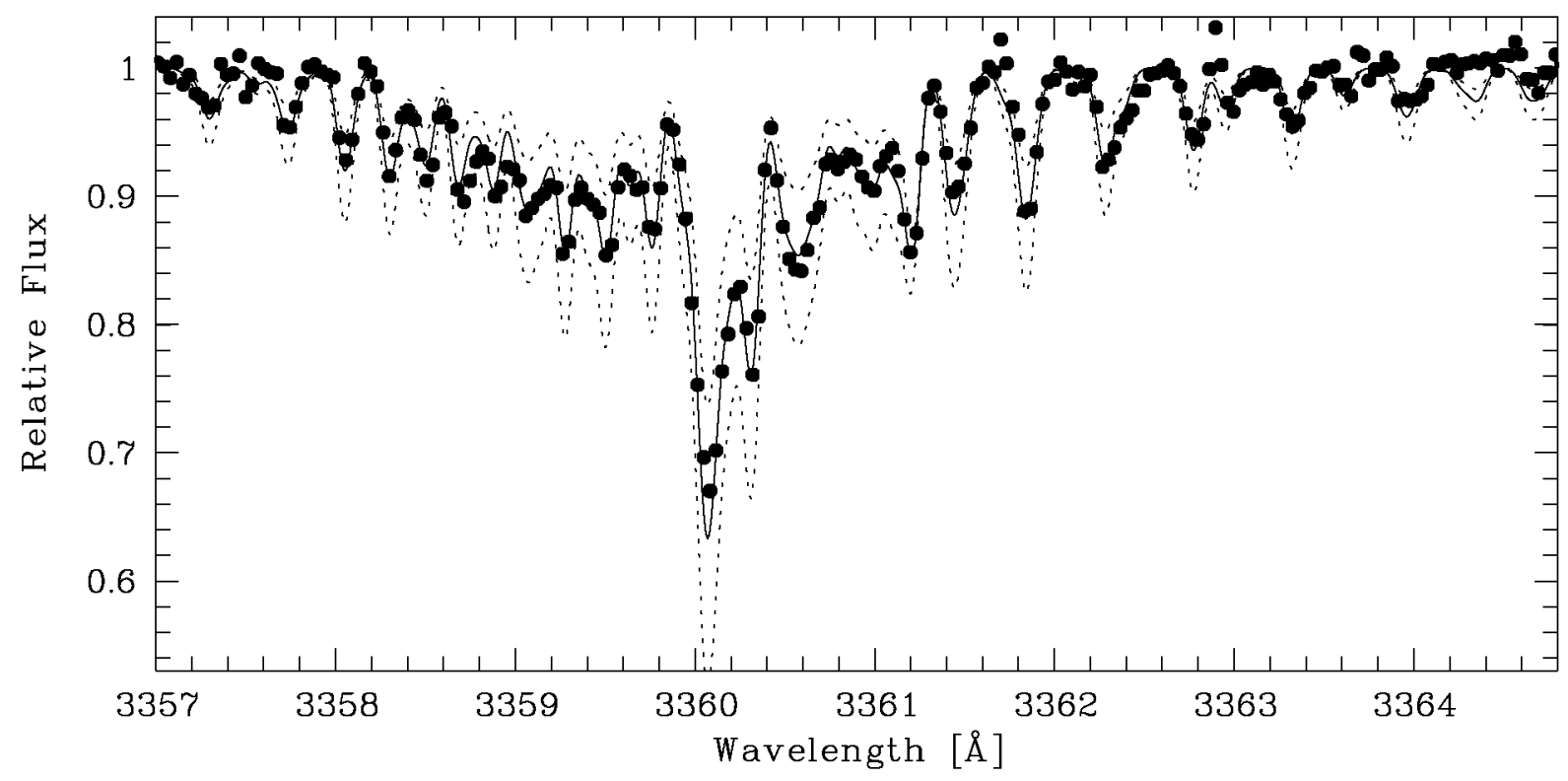

FIGURE 1. NH band spectral region. The observed spectrum (dots) is overplotted with "3D" synthetic spectra with $\mathrm{N}$ abundances of $[\mathrm{N} / \mathrm{Fe}]=4.1$ (solid line), 3.9, and 4.3 (dotted lines).

ent lines with a variety of excitation potentials. The 3D corrections depend on the excitation potentials of these contributing lines, thus, the "final" correction has to be a blend of 3D corrections derived for individual lines. Collet et al. [8] computed 3D corrections for a few fictitious lines of $\mathrm{CH}, \mathrm{NH}$, and $\mathrm{OH}$ with selected excitation potentials. By using fictitious lines, the $\mathrm{CNO} 3 \mathrm{D}$ correction could be studied without a spectrum synthesis with a 3D model atmosphere.

In order to apply the $3 \mathrm{D}$ corrections to the $\mathrm{CNO}$ abundances in a more suitable way, we anticipate a new technique. We first interpolate in between the given $\log \varepsilon 3 \mathrm{D}$ corrections to obtain appropriate corrections for all lines that comprise the molecular feature of interest. Then, abundance changes corresponding to the $3 \mathrm{D}$ corrections are applied to the $\log g f$ values for all lines in our linelist. The observed spectrum is then synthesized with the new "3D" linelist. Figure 1 shows the result for the NH band synthesis. The average $3 \mathrm{D}$ corrections are $\sim-0.7$ for all three CNO elements.

In the case of $\mathrm{N}$, irrespective of any resulting abundance, the use of the "3D" linelist results in an improvement of the new fit to the observed NH spectral region compared with the "1D" linelist fit. This effect cannot be "fixed" with just a simple abundance offset. The improvement results from different corrections on the red and blue side of the $\mathrm{NH}$ main feature at $3360 \AA$ based on the excitation potentials of the participating molecular lines.

\section{Lithium}

The Li I doublet at $6707 \AA$ is still not detected in this relatively unevolved star. From the very high $S / N(\sim 600$ per pixel) data we are able to derive a new $3 \sigma$ upper limit of $\log \varepsilon(\mathrm{Li})=A(\mathrm{Li})<0.7$ from our $1 \mathrm{D}$ analysis; this is further reduced to $A(\mathrm{Li})<0.6$ by application of a $3 \mathrm{D}$ correction. Figure 2 shows the Li spectral regions overplotted with synthetic spectra of different abundances. This is significantly lower than the previous value of $\log \varepsilon(\mathrm{Li})=1.6$ [3]. Other unevolved metal-poor stars with $[\mathrm{Fe} / \mathrm{H}] \sim-3.5$ have $\mathrm{Li}$ values around $\log \varepsilon(\mathrm{Li}) \sim$ 2.0 (e.g. Ryan et al. [10]). These objects have been used to infer the primordial Li abundance, which can be compared to the value expected from the baryon-to-photon ratio obtained by WMAP [11], $\log \varepsilon(\mathrm{Li})=2.6$. The discrepancy between the WMAP result and the metal-poor star values has amply been discussed, and we shall not further elaborate on it here. It is surprising, however, that the upper limit of HE 1327-2326 is not only lower than the WMAP result, but also considerably lower (by more than 1 dex) than values derived for other metal-poor stars with similar evolutionary status. This means that either all of this star's Li was destroyed during its lifetime on the main-sequence, or the star was born from Li-depleted material (see Piau et al. [12] for additional details).

The new 1D abundance analysis of HE 1327-2326, based on the currently best available observational data obtained with VLT/UVES, has been combined with 


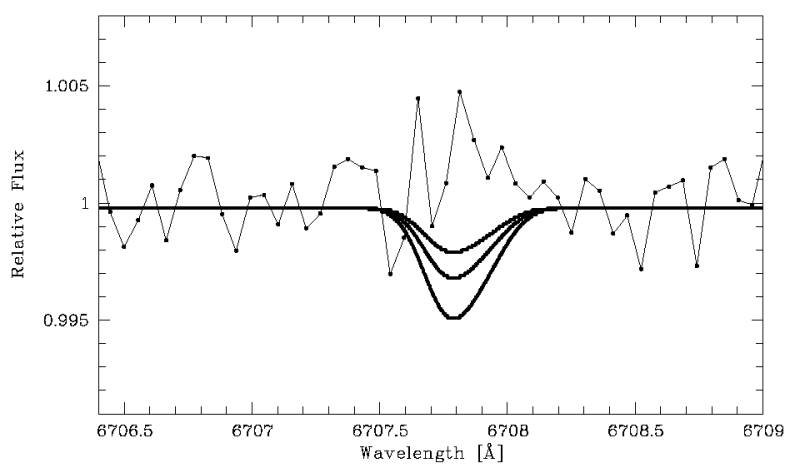

FIGURE 2. Spectral region (connected dots) of the Li line at $6707 \AA$ in HE $1327-2326$. Synthetic spectra with abundances of $\log \varepsilon(\mathrm{Li})=0.4,0.6$ and 0.8 are overplotted (solid lines).

state-of-the-art 3D model atmosphere corrections of Collet et al. [8]. These new 3D-corrected abundance measurements (for more details, see Frebel et al. [4]) are providing the basis on which the current models of the chemical yields of the first-generation SN can observationally be tested (e.g., Iwamoto et al. [13], Meynet et al. [14]).

\section{THE SAMPLE OF BRIGHT METAL-POOR STARS}

\section{New Analysis of the Med.-Resolution Data}

Frebel et al. [1] recently discussed observations of a sample of bright $(10<B<14)$ metal-poor stars identified in the Hamburg/ESO survey. The available mediumresolution data was analyzed for metallicity estimates, $[\mathrm{Fe} / \mathrm{H}]$, by means of the CaII $\mathrm{K}$ index and a color, and using the Beers et al. [15] calibration. Unfortunately, the stellar parameters $\left(\mathrm{T}_{\text {eff }}, \log g\right.$, and $\left.[\mathrm{Fe} / \mathrm{H}]\right)$ remained unknown from such an analysis. While the color served as temperature indicator, no information could be obtained for the surface gravity. However, knowledge of the surface gravity is crucial for deriving stellar distances, and hence for any kinematic analyses.

Lee et al. [5, 6] have recently developed an extensive software pipeline (the SSPP) for obtaining estimates of the atmospheric parameters of stars with mediumresolution spectroscopy obtained during the course of the SDSS, and its extension (SDSS-II, which includes the program SEGUE: Sloan Extension for Galactic Understanding and Exploration). From a pipeline addition (Marsteller et al. 2007, in prep.), [C/Fe] abundance ratios are also derived. We refer the interested reader to these papers for detailed descriptions of the pipelines. We have used this new, powerful tool to re-analyze our entire sam- ple of bright metal-poor stars. Frebel et al. [1] were not able to assign metallicity estimates to the more metalrich stars $([\mathrm{Fe} / \mathrm{H}]>-1.0)$ due to insensitivity of their analysis techniques.

With the new pipeline, stars at any metallicity can be analyzed. We thus obtain stellar parameters of the entire sample, which spans the metallicity range from $[\mathrm{Fe} / \mathrm{H}]=-5.1$ to +0.5 . The temperature range covered by SSPP is 3500 to $7500 \mathrm{~K}$ (a few bright stars that exceed the upper limit have been excluded). The vast majority of the metal-rich stars appear to be main-sequence stars, while the more metal-poor ones are subgiants and giants. Distances and $U V W$ space motions are then obtained.

\section{Galactic Populations}

Based on SDSS stellar data, Carollo et al. [16] recently found a clear signature of two different halo populations (the "inner" and "outer" halo) with net prograde and retrograde motions, and different peaks in their metallicity distribution functions, respectively.

Figure 3 shows $U$ and $V$ velocities as a function of $[\mathrm{Fe} / \mathrm{H}]$ for both the bright-star and the HERES samples Barklem et al. [17]. See also Beers et al. (this volume) for a comparison to the SDSS sample. The majority of the bright stars are located between $[\mathrm{Fe} / \mathrm{H}] \sim 0$ and $\sim-1$ and exhibit $U V W$ velocities around $0 \mathrm{~km} / \mathrm{s}$. These stars can be associated with the thick disk population. Overplotted with circles are all the bright stars that had metallicities assigned by Frebel et al. [1]. The stars that have space velocities not consistent with the bulk of the thick disk (and similarly with the metal-weak tail of the thick disk), can be considered halo members. It can be seen that the majority of these halo stars had been successfully picked up as metal-poor stars by Frebel et al. [1] despite their limited analysis possibilities. Furthermore, overplotted with crosses are the stars from the HERES sample. These objects are almost all halo members, as could be expected from their low metallicities $(-3.7<[\mathrm{Fe} / \mathrm{H}]<-1)$ and fainter magnitudes.

\section{The Frequency of Carbon-Enhanced Metal-Poor Stars}

Understanding the reasons for the large numbers of Carbon-Enhanced Metal-Poor (CEMP) stars found among metal-poor samples with $[\mathrm{Fe} / \mathrm{H}]<-2.0$, and the corresponding frequency of such objects as a function of metallicity, is currently an active area of research. Table 1 lists the results of several recent studies found in the literature. 

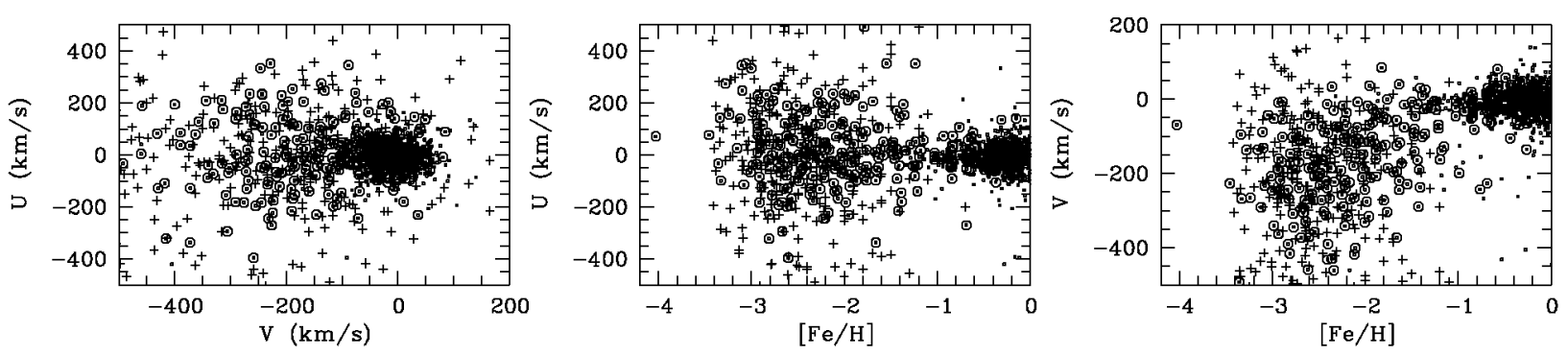

FIGURE 3. $U$ and $V$ velocities as a function of $[\mathrm{Fe} / \mathrm{H}]$. Small dots indicate bright HES stars and crosses show those stars for which Frebel et al. [1] were able to assign metallicities. Circled dots are HERES stars [17, 18].

TABLE 1. Frequency of C-rich stars as found in the literature. Note that the Marsteller et al. value is for $[\mathrm{Fe} / \mathrm{H}<-2.5$.

\begin{tabular}{|c|c|c|}
\hline Sample & $\begin{array}{l}{[\mathbf{F e} / \mathbf{H}]} \\
<-2.0\end{array}$ & Comment \\
\hline $\begin{array}{r}\text { Marsteller et al. [20] } \\
\text { Cohen et al. [21] } \\
\text { Lucatello et al. [18] } \\
\text { Lai et al. [22] }\end{array}$ & $\begin{array}{r}25 \% \\
14 \% \\
>21 \% \\
8-18 \% \\
\end{array}$ & $\begin{array}{l}\text { giants }+ \text { dwarfs } \\
\text { faint HES giants } \\
\text { faint HES giants } \\
\text { giants }+ \text { dwarfs }\end{array}$ \\
\hline $\begin{array}{r}\text { Frebel et al. [1] } \\
\text { Frebel et al. (in prep.) }\end{array}$ & $\begin{array}{r}9 \% \\
27 \%\end{array}$ & $\begin{array}{l}\text { bright HES giants } \\
\text { same as above }\end{array}$ \\
\hline
\end{tabular}

Frebel et al. [1] reported a lower value of $9 \%$ based the analysis of the CaIIK and CH G-band indices and the regressions given by Rossi et al. [19]. As an additional part of the new SSPP analysis, the G-band region is synthesized to obtain estimates of the C abundances (B. Marsteller et al. 2007, in prep). With no distinction between giants or dwarfs we preliminarily find a frequency of $27 \%$ among stars with $[\mathrm{Fe} / \mathrm{H}]<-2.0$. For giants only, we derive $12 \%$, while $62 \%$ for dwarfs (which is based on only a few stars). The distinction is made by a temperature cut at $5500 \mathrm{~K}$.

The previous lower value of $9 \%$ among the subsample of metal-poor giants can be understood as follows. As an initial gravity indicator, a simple color cut was applied (due to the limitations of the analysis technique, no better choice could have been made). However, the available colors were subject to, in some cases, significant uncertainties (see also Frebel et al. [1] for more details on the uncertainties). This may have had an effect on the parent sample selection. Furthermore, the carbon abundances were based on a simple regression [19], that does not take into account the temperature of the stars. This may well have had an effect on the number of CEMP stars reported in a given subsample.

Still, there remains a significant variation of CEMP frequencies found by the different studies. This may have an underlying cause, if, for example, the different samples consist of members from both the inner- and outer- halo populations, and also have different evolutionary status. Small number statistics have almost certainly played a role as well. The bright-star sample, combined with the HERES sample, will be used to study these issues in the near future, which should shed some additional light on the origin of CEMP stars.

Frebel et al. [1] also suggested that the frequency of CEMP stars increases with increasing distances from the Galactic plane, between 0 and $5 \mathrm{kpc}$. Our new analysis indicates that this claim has to be revisited (Frebel et al. 2007 , in prep.).

\section{CEMP Stars as Cosmological Tools}

A very large fraction of (long-lived, low-mass) CEMP stars are found to be in binary systems [18]. Their erstwhile intermediate- mass companions reflect an underlying initial mass function in the range $1-8 \mathrm{M}_{\odot}$. Tumlinson [23] predicts that the fraction of CEMP stars should increase with declining metallicity, and that it should vary spatially at the same metallicity. The new increased CEMP fraction of $27 \%$ agrees much better with his prediction (see his Figure 2).

\section{APPLICATION OF NEW DATA TO STELLAR ARCHAEOLOGY}

Fine-structure line cooling through $\mathrm{C}$ and $\mathrm{O}$ may be responsible for the cooling of the interstellar medium in the early Universe, which led in turn to the formation of the first low-mass stars [24]. We have further populated the plot of Frebel et al. [25] with lower limits for $D_{\text {trans }}$, based on the new $[\mathrm{Fe} / \mathrm{H}]$ and $[\mathrm{C} / \mathrm{Fe}]$ data from the bright-star sample. For a low-mass star that is still observable today, it has to lay above the critical metallicity limit of $D_{\text {trans }}=-3.5$. As can be seen from Figure 4, a few stars lay in the "forbidden zone" with $D_{\text {trans }}<-3.5$. However, we strongly caution that these numbers have been derived from medium-resolution data alone, and 


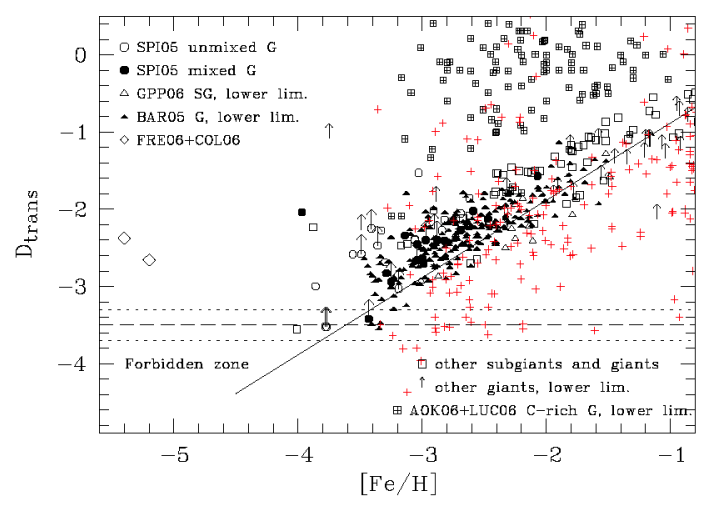

FIGURE 4. Updated plot from Frebel et al. [25] for the galactic halo, now with additional, new metal-poor data (lower limits) from the bright sample (crosses).

still have large uncertainties. For the majority of those few apparently carbon-deficient stars, we already have high-resolution observations available, and an investigation will be carried out in due course. We encourage observers to add their $\mathrm{C}$ and/or $\mathrm{O}$ measurements of metalpoor stars to such a plot in order to fully map the observable regions, and to test the fine-structure line theory of [24] with the most solid observational material.

\section{ACKNOWLEDGMENTS}

A. F. warmly thanks the conference organizers for a stimulating meeting, as well as for financial support. She gratefully acknowledges support through the W. J. McDonald Fellowship of the McDonald Observatory. T. C. B acknowledges support from grants AST 04-06784, AST 07-07776, and PHY 02-16783, Physics Frontier Centers/JINA: Joint Institute for Nuclear Astrophysics, awarded by the U.S. National Science Foundation.

\section{REFERENCES}

1. A. Frebel, N. Christlieb, J. E. Norris, T. C. Beers, M. S. Bessell, J. Rhee, C. Feehner, B. Marsteller, S. Rossi, C. Thom, L. Wisotzki, and D. Reimers, ApJ 652, 1585-1603 (2006).

2. A. Frebel, W. Aoki, N. Christlieb, H. Ando, M. Asplund, P. S. Barklem, T. C. Beers, K. Eriksson, C. Fechner, M. Y Fujimoto, S. Honda, T. Kajino, T. Minezaki, K. Nomoto, J. E. Norris, S. G. Ryan, M. Takada-Hidai, S. Tsangarides, and Y. Yoshii, Nature 434, 871-873 (2005).

3. W. Aoki, A. Frebel, N. Christlieb, J. E. Norris, T. C. Beers, T. Minezaki, P. S. Barklem, S. Honda, M. Takada-Hidai, M. Asplund, S. G. Ryan, S. Tsangarides, K. Eriksson, A. Steinhauer, C. P. Deliyannis, K. Nomoto, M. Y.
Fujimoto, H. Ando, Y. Yoshii, and T. Kajino, ApJ 639 897-917 (2006).

4. A. Frebel, N. Christlieb, K. Eriksson, and W. Aoki, ApJ, submitted (2007).

5. Y. S. Lee et al., AJ, submitted (2007)

6. Y. S. Lee et al., $A J$, submitted (2007)

7. A. Frebel, N. Christlieb, J. E. Norris, W. Aoki, and M. Asplund, ApJ 638, L17-L20 (2006).

8. R. Collet, M. Asplund, and R. Trampedach, ApJ 644 , L121-L124 (2006)

9. M. Asplund, $A A R \& A$ 43, 481-530 (2005).

10. S. G. Ryan, J. E. Norris, and T. C. Beers, $A p J \mathbf{5 2 3}$, 654-677 (1999)

11. D. N. Spergel, R. Bean, O. Doré, M. R. Nolta, C. L. Bennett, J. Dunkley, G. Hinshaw, N. Jarosik, E. Komatsu, L. Page, H. V. Peiris, L. Verde, M. Halpern, R. S. Hill, A. Kogut, M. Limon, S. S. Meyer, N. Odegard, G. S. Tucker, J. L. Weiland, E. Wollack, and E. L. Wright, ApJS 170, 377-408 (2007).

12. L. Piau, T. C. Beers, D. S. Balsara, T. Sivarani, J. W. Truran, and J. W. Ferguson, ApJ 653, 300-315 (2006).

13. N. Iwamoto, H. Umeda, N. Tominaga, K. Nomoto, and K. Maeda, Science 309, 451-453 (2005).

14. G. Meynet, S. Ekström, and A. Maeder, $A \& A 447$, 623-639 (2006).

15. T. C. Beers, S. Rossi, J. E. Norris, S. G. Ryan, and T. Shefler, $A J 117,981-1009$ (1999).

16. D. Carollo, T. C. Beers, Y. S. Lee, M. Chiba, J. E. Norris, R. Wilhelm, T. Sivarani, B. Marsteller, J. A. Munn, C. A. L. Bailer-Jones, P. Re Fiorentin, and D. G. York, astro-ph 0706.3005 (2007).

17. P. S. Barklem, N. Christlieb, T. C. Beers, V. Hill, M. S. Bessell, J. Holmberg, B. Marsteller, S. Rossi, F.-J. Zickgraf, and D. Reimers, $A \& A$ 439, 129-151 (2005).

18. S. Lucatello, T. C. Beers, N. Christlieb, P. S. Barklem, S. Rossi, B. Marsteller, T. Sivarani, and Y. S. Lee, $A p J$ 652, L37-L40 (2006).

19. S. Rossi, T. C. Beers, C. Sneden, T. Sevastyanenko, J. Rhee, and B. Marsteller, $A J$ 130, 2804-2823 (2005)

20. M. Marsteller, T. C. Beers, S. Rossi, N. Christlieb, M. S. Bessell, and J. Rhee, "Carbon-Enhanced Metal-Poor Stars in the Early Galaxy," in Nucl. Phys. A758, 2005, p. 312.

21. J. G. Cohen, S. Shectman, I. Thompson, A. McWilliam, N. Christlieb, J. Melendez, F.-J. Zickgraf, S. Ramírez, and A. Swenson, $A p J$ 633, L109-L112 (2005).

22. D. K. Lai, M. Bolte, J. A. Johnson, and S. Lucatello, $A J$ 128, 2402-2419 (2004).

23. J. Tumlinson, $A p J$ 664, L63-L66 (2007)

24. V. Bromm, and A. Loeb, Nature 425, 812-814 (2003).

25. A. Frebel, J. L. Johnson, and V. Bromm, MNRAS p. L71 (2007). 